\title{
Bardes de l'Himalaya. Epopées et Musique de Transe
}

Textes et enregistrements de Franck Bernède. 1998

\section{Riccardo Canzio}

\section{(2) OpenEdition}

Journals

Édition électronique

URL : http://journals.openedition.org/ethnomusicologie/1691

ISSN : 2235-7688

Éditeur

ADEM - Ateliers d'ethnomusicologie

Édition imprimée

Date de publication : 1 janvier 1998

Pagination : 304-306

ISBN : 2-8257-0639-6

ISSN : $1662-372 X$

Référence électronique

Riccardo Canzio, «Bardes de l'Himalaya. Epopées et Musique de Transe », Cahiers d'ethnomusicologie [En ligne], 11 | 1998, mis en ligne le 07 janvier 2012, consulté le 24 avril 2019. URL : http:// journals.openedition.org/ethnomusicologie/1691

Ce document a été généré automatiquement le 24 avril 2019

Tous droits réservés 


\section{Bardes de l'Himalaya. Epopées et Musique de Transe}

Textes et enregistrements de Franck Bernède. 1998

Riccardo Canzio

\section{RÉFÉRENCE}

Bardes de l'Himalaya. Epopées et Musique de Transe, Textes et enregistrements de Franck Bernède. 1 CDCNR 2741080, 1998 [Le Chant du Monde, Collection du CNRS et du Musée de L'Homme]

1 Quand on évoque les musiques de l'Inde, on pense rarement aux Himalayas. C'est aussi le cas pour le Népal, on pense à la vallée de Kathmandu ou aux marches tibétaines. Ainsi la région dont ce disque nous montre les richesses musicales (Garhwal et Kumaon du coté indien et l'extrême ouest du Népal) est encore peu connue, même des spécialistes, et cependant elle présente un intérêt ethnographique et musical exceptionnel. D'une grande unité culturelle (qu'elle hérite de l'ancien empire Malla qui, du XII ${ }^{e}$ au XIV ${ }^{e}$ siècle couvrait ce territoire), elle comporte des spécificités qui la démarquent du reste tant du Népal que des régions voisines au nord (notamment par l'absence des populations bhotia d'origine tibéto-birmane).

2 Nous y trouvons des pratiques religieuses sous-tendues par des rituels où la musique joue un rôle fondamental. C'est à travers une étude détaillée de ces pratiques qu'on pourra accéder à une connaissance plus solide de la culture de cette région. La musique y jouit d'une importance certaine, devenant ainsi un véritable outil d'investigation ethnologique dont toute étude sérieuse de cette aire culturelle ne saurait se passer.

Il est clair que l'auteur n'a pas eu l'intention de faire un disque à l'écoute facile car nous sommes confrontés à une musique très imbriquée dans son contexte. Elle exige de l'auditeur une connaissance approfondie des conditions de la performance s'il veut pouvoir en dégager la véritable signification. Cette production sonore possède à la fois 
une indéniable valeur esthétique et un intérêt musicologique qui dépasse le simple fait de sa singularité.

4 Le disque présente trois répertoires: les chants épiques, les musiques festives et les musiques cérémonielles liées à la transe. La notice donne un bref aperçu sur la vie et les sentiments d'une population relativement bien connue sur le plan ethnographique, mais demeurée largement ignorée des musicologues. L'ensemble des répertoires est indissociable du petit tambour-sablier à tension variable hudko, qui est l'instrument typique de cette région. Il semblerait pourtant qu'il fonctionne différemment dans les cérémonies de transe et les récits épiques que dans les circonstances festives. Dans ce dernier cas, ce sont les deux peaux du tambour qui sont battues, alors qu'aux occasions rituelles, seule la peau symbolisant les cieux est frappée.

5 Ces trois répertoires sont aussi associés aux différents rôles des musiciens. Le ménestrel et joueur de tambour (huḍkiya) est concerné essentiellement par l'aspect festif du répertoire, mais il peut être élevé au rang de barde (jagariya); il ne chantera alors que les récits épiques, mais pourra officier dans les cérémonies de transe en induisant la possession du médium (dangariya).

6 A propos de la transe, il faut mentionner les caractéristiques très particulières qu'elle revêt chez le chamane, qui agit comme un canal de communication avec les divinités locales, mais sans avoir accès au grandes divinités du panthéon classique hindou. Elle (parce qu'il s'agit plutôt d'une femme) est guidée par le barde dans son parcours rituel.

7 Le répertoire épique fait prendre conscience de la grande diversité stylistique rencontrée dans la région. Les deux rives de la rivière Mahakali (qui est la frontière politique entre l'Inde et le Népal) semblent aussi marquer une frontière stylistique: du point de vue théorique, la musique paraît beaucoup plus élaborée au Kumaon; on remarque en outre dans le disque de grandes différences de timbre et de traitement de la voix (couleurs vocaliques, techniques de vibrato, parlé-chanté, etc.) et on croirait percevoir de véritables lignages bardiques. La situation est évidemment complexe et une écoute attentive de la plage 2 suggère des relations stylistiques avec d'autres régions de l'Himalaya occidental.

8 Musicologiquement, ces variantes stylistiques pourront être vues comme des différences dans les pratiques du rite, qui évoluent à partir d'une structure centrale originelle. Nous ne postulons pas nécessairement une relation de cause à effet entre contexte culturel et système sonore, mais nous voyons le phénomème de variation stylistique dans une musique de ce type plutôt comme un processus où contexte et système sonore évoluent et se développent historiquement ensemble.

9 Il paraîtrait qu'autrefois, une des fonctions du barde était de réciter les généalogies des petits seigneurs. Aujourd'hui on assiste à une fusion des rôles du barde et du ménestrel, les fonctions de ces personnages ayant probablement été dissociées au temps des petits royaumes qui fleurissaient autrefois dans ce vaste territoire montagneux. Une perspective diachronique associée à une meilleure connaissance de ces royaumes pourra peut-être expliquer les variantes trouvées dans des récits épiques et ainsi éclairer ces différences de style.

10 On remarque que les protagonistes rituels sont issus des classes defavorisées : ce sont des tailleurs de la caste des intouchables. Il a été suggéré que les cultes de possession sont liés à ces catégories de la population qui utilisent la transe pour exercer des pressions sur la société environnante en cas de pénurie. La continuité de ce type de rite ne serait-elle pas 
un refuge pour des groupes plus ou moins marginalisés qui s'attachent à des valeurs constituant encore une composante importante de l'identité culturelle de cette société ?

11 Pour conclure, on pourrait dire que les pratiques qui sous-tendent la musique présentée dans ce disque présentent un véritable défi d'interprétation sur deux plans. Tout d'abord sur le plan ethnologique, le rôle multiple du meneur doit être clarifié - il est officiant dans des cérémonies de transe, il anime des festivités laïques, mais il est aussi barde et dépositaire des traditions orales. On remarque une série de significations étroitement liées au contexte de la transe telles que cris, gestes et autres signes, qu'il faut pouvoir décrire et interpréter pour expliquer le processus suposé ou réel de transpersonnalisation des médiums. Deuxièmement et plus fondamentalement, sur le plan musical, cette musique résiste à une analyse conventionnelle et probablement aussi à une transcription musicale conventionnelle. Ces deux aspects ne devront pas être négligés par la recherche si l'on veut parvenir à cette vision totale de la musique qui est l'objectif des efforts du musicologue et si l'on veut dégager des concepts qui pourront contribuer aux débats théoriques de l'ethnomusicologie. Franck Bernède nous convie à l'audition d'un monde musical et esthétique d'une beauté austère. Ce sera éventuellement sa tâche de nous guider et de nous aider à mieux comprendre cet univers qu'il nous offre aujourd'hui.

Les enregistrements originaux sont de très bonne qualité ; mais l'édition et le montage enlèvent l'éclat qu'on aurait pu attendre si des techniques modernes de post-production avaient été appliquées à la réalisation de ce disque. La notice informative est bien documentée, comme on peut s'y attendre de la part d'une collection qui donne aux chercheurs l'occasion de présenter le produit de leurs travaux indépendamment de toute considération commerciale. Sur ce point, cette collection doit être félicitée et encouragée à continuer dans la même voie. Pourtant, une mise en page peu professionnelle du livret, une mauvaise reproduction des photos en noir et blanc et une coquille dans le texte du titre sur la couverture ne sont pas dignes d'une collection qui prétend à la qualité.

La parution de ce disque intervient au moment où se manifeste un regain d'intérêt pour les musiques moins connues de cette région. Son importance ne réside pas seulement dans la redécouverte (pour la plupart des auditeurs il s'agira simplement d'une découverte) d'une musique d'un grande valeur esthétique et ethnographique; il contribue aussi à ouvrir un terrain de recherche très prometteur à l'intérieur du champ des études himalayennes, venant ainsi combler une lacune dans la discographie. Il s'agit d'une publication indispensable pour tous ceux qui sont concernés de près ou de loin par les musiques et les cultures de cette aire et du sous-continent indien de façon générale. 